\title{
Abnormal GABAergic Function and Negative Affect in Schizophrenia
}

\author{
Stephan F Taylor*, , Elise Demeter ${ }^{2}$, K Luan Phan ${ }^{3}$, Ivy F Tso ${ }^{4}$ and Robert C Welsh ${ }^{5}$ \\ 'Department of Psychiatry, University of Michigan, Ann Arbor, MI, USA; ${ }^{2}$ Department of Psychology, Duke University, Durham, NC, USA; \\ ${ }^{3}$ Department of Psychiatry, University of Illinois at Chicago and Mental Health Service, Jesse Brown Veterans Affairs Medical Center, Chicago, IL, USA; \\ ${ }^{4}$ Department of Psychology, University of Michigan, Ann Arbor, MI, USA; ${ }^{5}$ Department of Radiology, University of Michigan, Ann Arbor, MI, USA
}

\begin{abstract}
Deficits in the $\gamma$-aminobutyric acid (GABA) system have been reported in postmortem studies of schizophrenia, and therapeutic interventions in schizophrenia often involve potentiation of GABA receptors (GABAR) to augment antipsychotic therapy and treat negative affect such as anxiety. To map GABAergic mechanisms associated with processing affect, we used a benzodiazepine challenge while subjects viewed salient visual stimuli. Fourteen stable, medicated schizophrenia/schizoaffective patients and I 3 healthy comparison subjects underwent functional magnetic resonance imaging using the blood oxygenation level-dependent (BOLD) technique while they viewed salient emotional images. Subjects received intravenous lorazepam (LRZ; $0.01 \mathrm{mg} / \mathrm{kg}$ ) or saline in a single-blinded, cross-over design (two sessions separated by I-3 weeks). A predicted group by drug interaction was noted in the dorsal medial prefrontal cortex (dmPFC) as well as right superior frontal gyrus and left and right occipital regions, such that psychosis patients showed an increased BOLD signal to LRZ challenge, rather than the decreased signal exhibited by the comparison group. A main effect of reduced BOLD signal in bilateral occipital areas was noted across groups. Consistent with the role of the dmPFC in processing emotion, state negative affect positively correlated with the response to the LRZ challenge in the dmPFC for the patients and comparison subjects. The altered response to LRZ challenge is consistent with altered inhibition predicted by postmortem findings of altered GABAR in schizophrenia. These results also suggest that negative affect in schizophrenia/schizoaffective disorder is associated-directly or indirectly-with GABAergic function on a continuum with normal behavior.
\end{abstract}

Neuropsychopharmacology (2014) 39, 1000-1008; doi:I0.I038/npp.2013.300; published online 4 December 2013

Keywords: $\mathrm{fMRl}$; psychosis; emotion; benzodiazepine

\section{INTRODUCTION}

A solid body of evidence supports the involvement of $\gamma$ aminobutyric acid (GABA) systems in schizophrenia (Lewis et al, 2012). Postmortem studies have identified alterations in presynaptic components of GABAergic neurons, such as reductions in glutamic acid decarboxylase-67 required for GABA production, decreased GABA transporter-1 density, and alterations in postsynaptic GABA receptor (GABAR) binding/transcripts (recently reviewed in Nakazawa et al (2012). Consistent with these postmortem findings, pairedpulse transcranial magnetic stimulation has revealed reduced cortical inhibition (Daskalakis et al, 2002). However, functional imaging studies using ligands for the benzodiazepine receptor (Abi-Dargham et al, 1999) have

*Correspondence: Dr SF Taylor, Department of Psychiatry, University of Michigan, Rachel Upjohn Building, 4250 Plymouth Rd, Ann Arbor, MI 48109-2700, USA, Tel: +I 734936 4955, Fax: +I 734936 7868, E-mail: sttaylor@umich.edu

This work has been previously presented at the annual meeting of the Society of Biological Psychiatry, Philadelphia, PA, USA in May of 2012.

Received 24 June 2013; revised 6 October 2013; accepted 7 October 2013; accepted article preview online 24 October 2013 failed to show altered GABAR densities, whereas magnetic resonance spectroscopy studies of GABA concentrations have reported mixed results (Kegeles et al, 2012; Yoon et al, 2010). Thus, the functional significance of GABA alterations remains to be elucidated.

Work to date has focused on the role of GABA in cognitive dysfunction (eg, Menzies et al, 2007), based on the critical role of GABAergc interneurons in the inhibitoryexcitatory microcircuits associated with integrative activity such as gamma oscillations (Bartos et al, 2007). However, clinical experience suggests another possibility. Therapeutic manipulations of GABA, eg, with valproate and benzodiazepines, are commonly used as adjunctive treatments in schizophrenia (Wassef et al, 1999). Benzodiazepines can prevent a psychotic episode in the early stages of relapse (Carpenter et al, 1999), and blocking GABAR activity can induce psychotic symptoms in clinically stable schizophrenia patients (Ahn et al, 2011). Glutamic acid decarboxylase-67 abnormalities have also been identified in bipolar disorder (Guidotti et al, 2000) and GABAergic drugs are also used to improve mood regulation (Cousins and Young, 2007). Collectively, the clinical evidence suggests that explorations of emotion processing may be an important line of inquiry to uncover the potential 
functional significance of GABAergic abnormalities in schizophrenia.

Toward that end, we used lorazepam (LRZ) as a probe of GABAergic activity during an emotion evocation task to demonstrate that GABAergic abnormalities would be associated with greater negative affect in schizophrenia. LRZ is a non-subtype selective benzodiazepine, an allosteric modulator of GABAR that hyperpolarizes the cell and potentiates GABA function (Olsen and Tobin, 1990), and is commonly used to treat negative affect such as anxiety in psychosis (Wassef et al, 1999). Although emotional abnormalities in schizophrenia are typically studied as deficits in expression and hedonic capacity, persons with schizophrenia experience more affect than they express, report more negative affect in everyday life (Kring and Moran, 2008), and exhibit greater trait negative affect (Horan et al, 2008). Schizophrenia patients tend to appraise neutral and positive stimuli as more negative (Cohen and Minor, 2010), and their low threshold for perceived stress predicts poor social functioning independent of positive symptoms or cognitive function (Tso et al, 2010). Negative affect also appears in individuals at risk of psychosis before the development of psychotic symptoms (Phillips and Seidman, 2008). Overall, negative affect is an important dimension of psychosis (van Rossum et al, 2011), and we sought here to tie it with GABAergic dysfunction.

Our primary hypothesis focused on the medial frontal cortex (MFC; including anterior cingulate cortex (ACC) and medial prefrontal cortex $(\mathrm{mPFC})$ ), a region involved in emotion regulation (Etkin et al, 2011) and abnormally activated in schizophrenia during emotion tasks (Taylor et al, 2012). We also predicted an interaction with activity in the anterior insula (aIns), a region involved in emotional responses (Kober et al, 2008) and associated with benzodiazepine-induced blood oxygenation level-dependent (BOLD) effects on pain (Wise et al, 2007) and face emotion processing (Paulus et al, 2005). Based on postmortem work, we hypothesized that when subjects viewed salient emotional images, LRZ would have a reduced inhibitory effect on neurocircuits in these regions, which would be correlated with greater negative affect. We also predicted that group interactions with LRZ would be most prominent while processing negative stimuli based on our prior work showing abnormal responses to negatively valenced pictures in persons with schizophrenia (Taylor et al, 2011; Taylor et al, 2007).

\section{MATERIALS AND METHODS}

\section{Subjects}

Seventeen stable outpatients (Table 1) were recruited with DSM-IV schizophrenia or schizoaffective disorder (American Psychiatric Association, 1994) established by structured interview (First et al, 1995). All patients were without current depression, alcohol/substance abuse/ dependence ( $>12$ months without abuse/dependence), regular use of benzodiazepines (as needed usage permitted), current narcotic use, presence of a significant medical illness that could affect cerebral function or history of closed head injury. All subject assessment included clinical ratings by an experienced clinician (SFT) on the Brief
Psychiatric Rating Scale (Overall and Gorham, 1962), the Calgary Depression Scale (Addington et al, 1993), and the Scale for the Assessment of Negative Symptoms (Andreasen, 1983). To obtain a cohort of subjects with sufficient negative affect sensitive to LRZ manipulation, patients were required to score $\geqslant 3$ on the Brief Psychiatric Rating Scale anxiety rating. One subject withdrew from the first scan due to eyestrain and headache, and two additional subjects were later excluded for excessive movement, leaving 14 subjects (three with schizoaffective disorder) for analysis (for brevity, hereafter referred to as 'psychosis subjects'). Thirteen of the psychosis subjects were on stable doses of atypical antipsychotic medications, plus antidepressants $(n=2)$ and lamotrigine $(n=1)$.

Thirteen healthy comparison subjects (Table 1), without a lifetime history of Axis I psychiatric disorders (First et al, 1995), were recruited to match the age range, gender distribution and family education level of the patients. They were also without first-degree relatives with psychosis or other serious mental illness (requiring antipsychotic medication, hospitalization, or ECT) and not taking medication affecting neural activity.

Additional assessments for all subjects included the Wide Range Achievement Test, revised, Reading subtest (Jastak and Wilkinson, 1984) for general intellectual achievement and the Brief Assessment of Cognition for general neurocognition (Keefe et al, 2004). Before each scanning session, subjects completed self-report measures of emotional state: The Perceived Stress Scale (Cohen et al, 1983), the Spielberger State-Trait Anxiety (state; Spielberger et al, 1983), and the Differential Emotions Scale (DES, yielding sub-scales for negative (DES-neg) and positive (DES-pos) emotions; (Fredrickson et al, 2003).

Before data collection, subjects gave written, informed consent to participate in the protocol approved by the University of Michigan institutional review board.

\section{Task Design}

Three sets of 36 unique images were selected from the International Affective Picture System: Neutral (NEUT), positive (POS), and aversive (NEG), matched on the presence $(\sim 50 \%)$ of human figures (Supplementary Table S1). Subjects were instructed to 'Press the button when the picture changes and experience the feeling you get from each picture.' Pictures were displayed in blocks, grouped by valence, for $3 \mathrm{~s}$ each, four pictures/block. A gray screen with a fixation cross separated each block (11-12.4 s duration). Order of presentation was pseudo-randomized based on simulations to optimize experimental power.

Before the first session, all subjects viewed stimuli in a mock scanner to desensitize them to the MRI environment, followed by a rating task of the same International Affective Picture System stimuli used in the fMRI acquisition to reduce variance during the scanning sessions caused by exposure to novel stimuli. Thus, during both fMRI acquisitions, no stimuli were novel to the subjects.

\section{Scanning Sessions}

Subjects underwent two fMRI scanning sessions in a singleblinded, cross-over design. All subjects had negative urine 
toxicology screens for drugs of abuse before each scanning session. Four subjects used benzodiazepines as needed, but with no usage occurred within five half-lives of the MRI session except for one subject who had clonazepam $0.5 \mathrm{mg}$, 3 day before one scanning session. After placement of an intravenous line, they received bolus injections either of LRZ $0.01 \mathrm{mg} / \mathrm{kg}$ or an equivalent amount of saline (SAL) solution. The sessions occurred with a minimal separation of 3 days (mean 12.1 \pm 9.7 days) in counterbalanced order for LRZ and SAL. Subjects were placed in the MRI scanner and task acquisition began $\sim 30 \mathrm{~min}$ after injection, coinciding with peak blood levels of intravenous LRZ (Wermeling et al, 2001). Each session consisted of the International Affective Picture System task as well as another activation task not reported here, with task order counterbalanced within each group. Before intravenous line placement, before each task and after the last task, subjects completed six visual analog scales assessing their subjective feelings of drowsiness, anxiety, happiness, fear, sadness, and excitement.

\section{Functional MRI Acquisition}

MRI scanning occurred on a GE 3T Signa scanner (LX [8.3] release, General Electric Healthcare, Buckinghamshire, UK). A T1-weighted image was acquired in the same prescription as the functional images to facilitate co-registration. Functional images were acquired with a $\mathrm{T} 2{ }^{\star}$-weighted, reverse spiral acquisition sequence (gradient recalled echo, $\mathrm{TR}=2000 \mathrm{msec}, \mathrm{TE}=30 \mathrm{msec}, \mathrm{FA}=90$ degrees, field of view $=22 \mathrm{~cm}, \quad 40$ slice, $3.0 \mathrm{~mm}$ thick $/ 0 \mathrm{~mm}$ skip, and equivalent to $64 \times 64$ voxel grid-yielding isotropic voxels $3 \mathrm{~mm}$ on edge) sensitive to signal in ventral medial frontal regions (Yang et al, 2002). Subjects underwent three runs (nine blocks/run), each consisting of 115 volumes (including viewing a baseline fixation cross six volumes before the first block and seven volumes after the last block) for a total of 345 volumes. Four initial volumes were discarded to allow for equilibration of scanner signal. Total duration of the task was $\sim 12 \mathrm{~min}$. After acquisition of functional volumes, a high-resolution T1 scan (3D SPGR, field of view $=26 \mathrm{~cm}, \mathrm{~T} 1=500 \mathrm{~ms}, \mathrm{TR}=20 \mathrm{msec}, \mathrm{TE}=1.8 \mathrm{msec}$, $256 \times 256$ matrix, 124 slices, and $1.2-\mathrm{mm}$ interleaved with no skip) was obtained for anatomic normalization.

\section{fMRI Data Analysis}

Data processing began with standard pre-processing steps. All scans were realigned to the first image acquired during a scanning session (Jenkinson et al, 2002). Re-alignment parameters were inspected to ensure that movement did not exceed either 1 voxel or 2 degrees rotation within a run. Subsequent processing was performed with the Statistical Parametric Mapping SPM8 package (Wellcome Institute of Cognitive Neurology, London, UK). Anatomic normalization using the high-resolution T1 image was applied to the time series of co-registered, functional volumes, which were re-sliced and smoothed with a 6- $\mathrm{mm}$ isotropic Gaussian smoothing kernel. At the first level, time series from anatomically normalized images were high-pass filtered (128s), and modeled with regressors for each of the three picture valences (NEG, NEUT, and POS), crossed with the two sessions (LRZ and SAL) for each 12-second block along with six movement regressors. Regressors were convolved with a canonical hemodynamic response function at the subject level, yielding estimates for the magnitude of the hemodynamic response function. Beta estimates were spatially smoothed again with a $6-\mathrm{mm}$ Gaussian kernel to stabilize variance properties and taken to a second-level, flexible factorial analysis, with session, valence, and group as factors. Planned analyses were carried out to examine main effects of drug (session), group by drug by valence interactions and group by drug interactions.

Statistical inference was controlled using Gaussian random field theory (Worsley and Friston, 1995). Small volume corrections were applied with masks for MFC and bilateral aIns (Tzourio-Mazoyer et al, 2002). For display, an uncorrected threshold of $p<0.005$ and cluster size $>30$ was used, but for significance, a family-wise error corrected, peak-level threshold of $p<0.05$ was required.

\section{Behavioral Data Analysis}

The behavioral data was analyzed with SPSS, version 19, in mixed model ANOVAs, with group as a between-subjects factor and Greenhouse-Geisser corrections applied for nonsphericity.

\section{RESULTS}

\section{Behavioral Results}

There were no significant effects of LRZ on behavior and arousal and mild effects on reported mood. Only 8 of 14 $(p=0.59)$ psychosis subjects and 7 of $13(p=0.79)$ comparison subjects guessed correctly which session involved LRZ. LRZ reduced fearfulness $(\mathrm{F}(1,23)=4.8, p=0.04)$ and showed a trend to reducing anxiety $(\mathrm{F}(1,23)=3.49$, $p=0.07)$. Although group by drug interactions were not significant, the LRZ-induced reduction of anxiety and fearfulness appeared driven by the psychosis patients. The subjects' button press in response to each picture provided an index of attentiveness to the stimuli. Latency between and within groups showed no group effects, no drug effects, and no interactions. For complete statistics and additional behavioral data, see Supplementary Material, Supplementary Figure S1 and Supplementary Tables S2 and S3.

\section{Effects of LRZ on BOLD Signal}

LRZ had significant effects on BOLD signal, both between and within groups. Testing the hypothesis that psychosis patients would exhibit a more pronounced response to negative stimuli interacting with LRZ, no significant group by drug by valence interactions were found in the voxelwise search of the whole brain or in a priori regions. We also separately examined $t$-tests for the effects of LRZ on NEG > NEUT, as well as POS > NEUT, contrasts and found no significant effects in a priori regions or in wholebrain searches. However, the group by drug analysis for all valences together (relative to the implicit baseline) revealed foci in the primary a priori region (dorsal $\mathrm{mPFC}, \mathrm{dmPFC}$ ), but not in the aIns. Significant foci also appeared in the 
Table I Demographical and Clinical Characteristics of Subjects

\begin{tabular}{|c|c|c|c|c|}
\hline Mean \pm SD & $\begin{array}{l}\text { Psychosis } \\
\text { patients } \\
(n=14)\end{array}$ & $\begin{array}{l}\text { Healthy } \\
\text { control subjects } \\
(n=13)\end{array}$ & $t \cdot \chi^{2}$ & $p$-values \\
\hline \multicolumn{5}{|l|}{ Demographics } \\
\hline Age, years & $43.0 \pm 12.6$ & $41.5 \pm 12.7$ & 0.30 & 0.77 \\
\hline Males/females & $9 / 5$ & $8 / 5$ & 0.22 & 0.88 \\
\hline Parental education, years & $16.5 \pm 2.7$ & $14.9 \pm 2.8$ & 1.49 & 0.15 \\
\hline Subject education, years & $15.4 \pm 2.7$ & $16.3 \pm 2.1$ & 1.00 & 0.32 \\
\hline Socio-economic status ${ }^{\mathrm{a}}$ & $2.64 \pm 0.94$ & $2.92 \pm 0.76$ & 0.85 & 0.41 \\
\hline Duration ill, years & $23.8 \pm 14.3$ & & & \\
\hline Hospitalizations & $3.6 \pm 1.7$ & & & \\
\hline \multicolumn{5}{|l|}{ Neurocognitive assessments } \\
\hline WRAT- $R$ reading & $50.8 \pm 7.0$ & $53.5 \pm 2.3$ & 1.31 & 0.20 \\
\hline $\mathrm{BACS}^{\mathrm{b}}$ & $-1.55 \pm 1.47$ & $-0.1 \pm 1.0$ & 3.00 & 0.006 \\
\hline \multicolumn{5}{|l|}{ Emotion assessments ${ }^{c}$} \\
\hline PSS & $14.7 \pm 5.9$ & $8.9 \pm 5.0$ & 2.73 & 0.011 \\
\hline STAl & $33.0 \pm 9.1$ & $27.9 \pm 4.6$ & 1.81 & 0.083 \\
\hline DES-positive & $2.69 \pm 0.53$ & $2.96 \pm 0.77$ & -1.06 & 0.30 \\
\hline DES-negative & $1.07 \pm 0.50$ & $0.44 \pm 0.29$ & 3.96 & 0.001 \\
\hline \multicolumn{5}{|l|}{ Symptom assessments } \\
\hline BPRS total ${ }^{c}$ & $29.5 \pm 4.5$ & & & \\
\hline BPRS positive $e^{c}$ & $7.5 \pm 2.5$ & & & \\
\hline BPRS negative ${ }^{c}$ & $6.0 \pm 1.9$ & & & \\
\hline $\operatorname{CDS}^{c}$ & $2.1 \pm 2.4$ & & & \\
\hline SANS global sum & $4.9 \pm 2.6$ & & & \\
\hline
\end{tabular}

Abbreviations: BACS, Brief Assessment of Cognition in Schizophrenia; BPRS, Brief Psychiatric Rating Scale; CDS, Calgary Depression Scale; DES, Differential Scale of Emotions; PSS, Perceived Stress Scale; SANS, Scale for the Assessment of Negative Symptoms; STAI, Spielberger State-Trait Anxiety; WRAT-R, Wide Range Achievement Test, revised, Reading subtest.

aBased on Holingshead-Redlich scale (Hollingshead and Redlich, 1958).

${ }^{b}$ Reported as Z-scores normalized to performance of control subjects.

'Assessments averaged across both scanning sessions, Symptom and emotion assessments before each scanning session did not differ across the sessions (except BPRS total: $30.2 \pm 4.4$ for first session, $28.8 \pm 4.8$ for second session; $\mathrm{t}(\mathrm{I})=2.54, p=0.02)$

right superior frontal gyrus, and left and right occipital regions (Table 2 and Figure 1a). Extractions from these regions showed that LRZ, relative to the implicit baseline, decreased BOLD signal in the control subjects, whereas LRZ tended to increase BOLD signal in the patients (Figure 2a). Post hoc tests of the dmPFC focus revealed that this focus exhibited reduced activity during SAL in the psychosis patients $(t(25)=2.3, p=0.03)$.

An analysis of the main effect of drug showed multiple clusters in the occipital lobe and adjacent cerebellum (Figure $1 \mathrm{~b}$ and Table 2). Extractions of parameter estimates from the highest magnitude peak of the right and left occipital clusters (Figure $2 \mathrm{~b}$ ) showed that the effect was a reduction of BOLD signal by LRZ for both groups on the right side, but mainly in the control group on the left side. A post hoc group by drug interaction on the extracted data was significant for the left side $(\mathrm{F}(1,25)=6.3, p=0.02)$, but not the right $(\mathrm{F}(1,25)=3.0, p=0.10)$.

\section{Correlations with Negative Affect and LRZ-Induced BOLD Changes}

To test specific hypotheses about GABAergic involvement in negative affect, planned correlations were run between the dmPFC focus of LRZ-induced change (taking extracted parameter estimates of LRZ-SAL) and negative affect measures (DES-negative, Perceived Stress Scale, and Spielberger State-Trait Anxiety). Negative affect positively correlated with BOLD response to LRZ challenge in the dmPFC for psychosis patients and control subjects, such that more negative affect was associated with a greater increase (or less decrease) of BOLD signal in response to LRZ (Figure 3 and Supplementary Table S4). Furthermore, a greater reduction in visual analog scale-rated anxiety after LRZ infusion was also associated with greater BOLD increase in the dmPFC but only for the schizophrenia subjects $(r=0.62, p=0.023)$. There were no significant correlations with Brief Psychiatric Rating Scale total, positive or negative symptoms, although there was a correlation with clinicianrated Calgary Depression Scale scores in the psychosis subjects $(r=0.53, p=0.049)$, consistent with the negative affect measured by the self-report scales.

Correlations with antipsychotic dosages in chlorpromazine equivalents (Woods, 2003) were non-significant for all extracted regions ( $p$-values $>0.3$ ). In addition, inspection of scattergrams showed that neither antidepressant/mood stabilizer usage (three patients) nor a schizoaffective diagnosis (three patients) were driving the correlations.

\section{DISCUSSION}

As predicted, the psychosis patients exhibited an altered response to the GABAergic manipulation. Group by drug interactions were noted in the $\mathrm{dmPFC}$, predicted within the MFC as well as several other areas. In line with the focus on affect processing, state negative affect positively correlated with the response to the LRZ challenge in the dmPFC for the patients and comparison subjects. The study represents the first imaging study, of which we are aware, in which a GABAergic manipulation is associated with affect in psychosis, with implications for the functional interpretation of GABAergic systems in psychotic disorders.

Although we have no way of knowing whether or not the patients we scanned also exhibited GABAR abnormalities reported from postmortem samples, the differential response of the psychosis patients to LRZ in the group by drug interactions was consistent with postmortem results. Whereas control subjects showed a reduced BOLD signal after LRZ, the patients showed an increase in BOLD signal in regions with group by drug interactions. Although there are questions about the effect of potentiating GABAR on a macro-level signal such as the fMRI BOLD response, it is possible to advance informed speculation about our results. Postmortem work has identified downregulation of postsynaptic GABAR (predominantly the $\alpha-1$ subunit) on excitatory pyramidal cells in schizophrenia, the postsynaptic targets of GABAergic and parvalbumin-expressing basket cell interneurons (Lewis et al, 2012; Nakazawa et al, 2012). Thus, potentiating GABAR should lead to less inhibitory influence on pyramidal cells in psychosis patients compared with the healthy subjects. Another class of 
Table 2 Effects of Lorazepam on BOLD Signal

\begin{tabular}{|c|c|c|c|c|}
\hline Region $^{\mathrm{a}}$ & $(x, y, z)^{b}$ & Cluster size (voxels) & Peak Z & Probability (corrected) \\
\hline \multicolumn{5}{|l|}{ Group by drug interactions } \\
\hline dmPFC & $-3,53,25$ & 153 & 3.78 & $0.050^{c}$ \\
\hline R superior frontal g & $27,17,67$ & 241 & 4.61 & 0.037 \\
\hline \multirow[t]{2}{*}{$L$ fusiform g } & $-39,-67,-23$ & 1722 & 5.68 & 0.000 \\
\hline & $-33,-64,-17$ & d & 5.67 & 0.000 \\
\hline $\mathrm{R}$ cerebellum/fusiform $\mathrm{g}$ & $36,-52,-26$ & d & 5.28 & 0.002 \\
\hline \multicolumn{5}{|l|}{ Main effects of drug } \\
\hline \multirow[t]{2}{*}{ R middle/inferior occipital and middle/inferior temporal g } & $39,-76,4$ & 1554 & 6.17 & 0.000 \\
\hline & $39,-94,-2$ & d & 5.28 & 0.002 \\
\hline \multirow[t]{4}{*}{ L middle/inferior occipital $g$ and middle/inferior temporal $g$} & $-30,-94,-2$ & 1140 & 6.09 & 0.000 \\
\hline & $-33,-94,16$ & d & 5.42 & 0.001 \\
\hline & $-51,-79,-5$ & d & 5.39 & 0.001 \\
\hline & $-39,-64,-5$ & d & 4.79 & 0.017 \\
\hline L cerebellum & $-39,-70,-23$ & d & 5.38 & 0.001 \\
\hline
\end{tabular}

Abbreviations: BOLD, blood oxygenation level-dependent; g, gyrus; L, left; R, right.

a Regions from (Tzourio-Mazoyer et al, 2002),

bStereotactic coordinates from MNII 52 reference, left/right, anterior/posterior and superior/inferior, respectively.

'Small volume correction for a priori regions.

${ }^{\mathrm{d}}$ Peak voxel part of a single super-cluster.

a

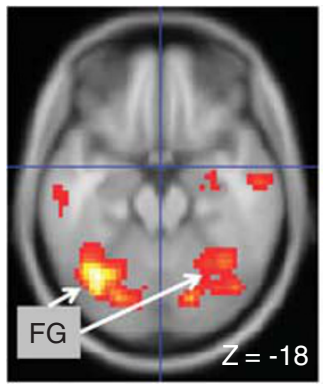

b

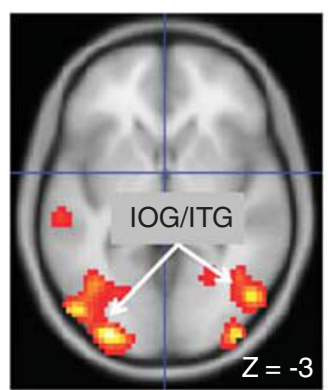

Group X drug interactions
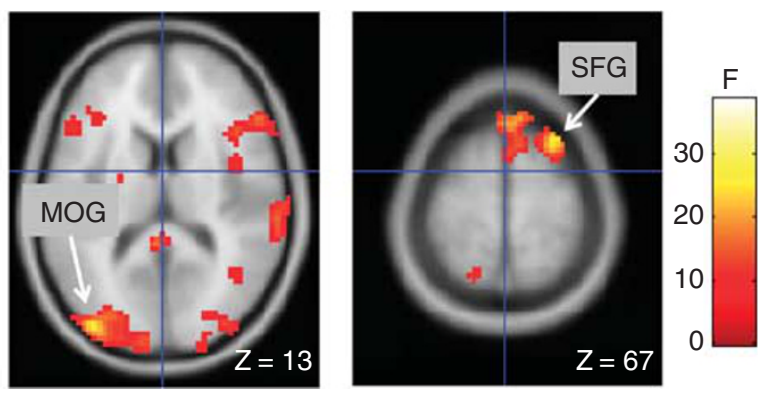

Main effects of drug
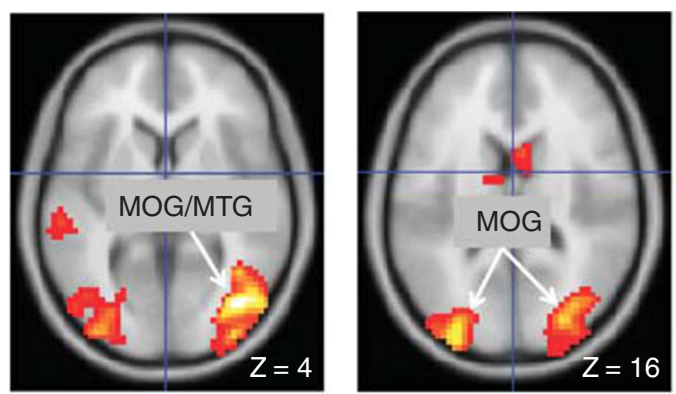

Figure I Effects of lorazepam (LRZ)on blood oxygenation level-dependent (BOLD) signal. (a) Group by drug interactions and (b) main effects of LRZ (relative to implicit baseline). Voxels rendered at uncorrected significance $p<0.005$; labels indicate significant foci with whole-brain correction. Right side of brain is on right side of panels. FG, fusiform gyrus; IOG, inferior occipital gyrus; ITG, inferior temporal gyrus; MOG, middle occipital gyrus; MTG, middle temporal gyrus; SFG, superior frontal gyrus. 

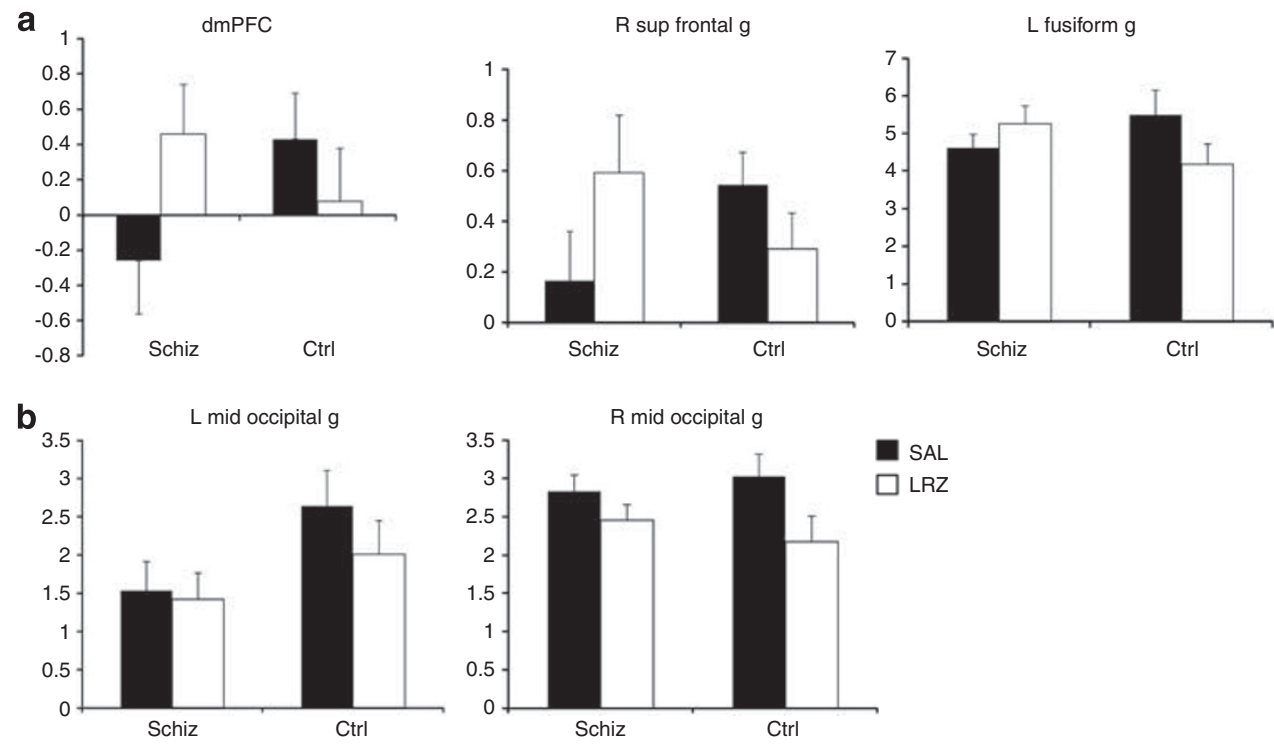

Figure 2 Extractions of parameter estimates of peak foci for group by drug interactions (a) demonstrate the effects of lorazepam (LRZ) reducing blood oxygenation level-dependent (BOLD) signal in the healthy control subjects (Ctrl), whereas increasing it for the psychosis subjects (Schiz). Extractions from foci showing main effects of drug (b) show similar reductions of BOLD signal in the right middle occipital gyrus, but less in the Schiz subjects for the left middle occipital gyrus. Ordinate on all graphs indicates parameter estimates in arbitrary units ( \pm SEM).
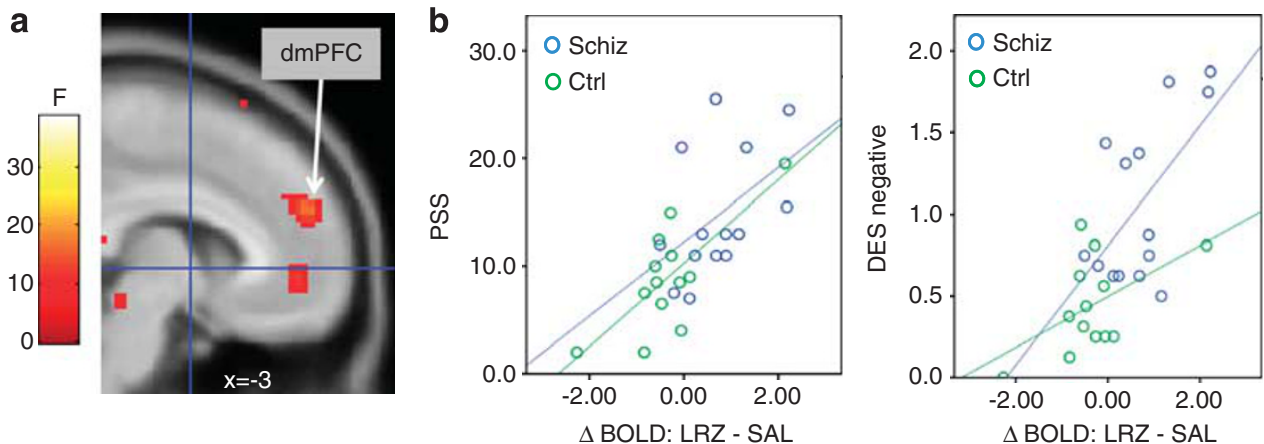

Figure 3 (a) The dorsal medial prefrontal cortex (dmPFC) focus indicates the predicted group by drug interaction. (b) Parameter estimates were extracted from the dmPFC cluster of the predicted group by drug interaction after lorazepam (LRZ) challenge (cluster with peak at - 3, 53, and 25), showing correlations with negative affect measured by Perceived Stress Scale (PSS; psychosis patients: $r=0.47, p=0.09$; comparison subjects: $r=0.74$, $p=0.004$ ) and Differential Emotions Scale (DES)-negative (psychosis patients: $r=0.60, p=0.02$; comparison subjects: $r=0.52, p=0.07$ ).

parvalbumin + interneurons, the chandelier cells, synapse on the spike-initiating segment of the axon of pyramidal neurons, and studies have revealed upregulated postsynaptic GABAR (predominately containing the $\alpha$-2 subunit) associated with these interneurons (Volk et al, 2002). In contrast to basket cells, chandelier cells appear to depolarize the axon, leading to excitation in rodent studies (Szabadics et al, 2006; Woodruff et al, 2009). Thus, if the rodent model can be applied to humans, GABAR potentiation would lead to greater excitation of pyramidal cells in schizophrenia via the chandelier cells. Taking these two postmortem GABAR findings together-less inhibition and greater excitationthe combined effect would increase BOLD signal after LRZ challenge, which is what we found.

The observed correlations between affect measures and LRZ effect in the dmPFC provide some clues about GABAergic dysfunction. We hypothesized that effects of
LRZ would occur in regions involved in affect processing during the task, hence our focus on the MFC. In the dmPFC, a region involved in emotion appraisal and expression (Etkin et al, 2011), activity during SAL was reduced in the patients, consistent with reduced activity reported in schizophrenia in this region when processing socio-emotional stimuli (Taylor et al, 2012). The association between LRZ-induced BOLD changes and negative affect appeared across multiple different scales at $p<0.05$ or trend levels: all three measures of self-rated state negative affect for the psychosis subjects and in two for the comparison subjects. The significant relationship in the control group suggests a pathophysiological process that is continuous from health through psychosis. This association-in the unmedicated healthy subjects-cannot be attributed to medication effects, which suggests that medication effects are also not driving the association in the patients. 
The correlation between reduced anxiety with LRZ and increased dmPFC BOLD signal in patients may seem counter-intuitive if one assumes that reduced GABAR inhibition means less capacity to reduce negative affect. However, it is important to recognize that the LRZ challenge uncovered aberrant activity in a dynamic system, possibly reflecting an imbalance between cortical excitation and inhibition (Lewis et al, 2012). Individuals with this imbalance may have a greater response to GABAR potentiation because they benefit more from potentiation of the reduced inhibitory function of the system, consistent with the clinical observation that some psychosis patients seek out benzodiazepines. The relationship was not seen with the comparison group, but they had significantly less negative affect overall, thus smaller variance in anxiety change scores. The effect of benzodiazepines on GABAR may not be directly related to negative affect; another mechanism-eg, glutamatergic dysfunction-may be the direct mediator of negative affect. Fully testing these interpretations will require additional research, but broadly speaking, the results are in line with a dimensional construct of negative affect, increasingly recognized as one important component of schizophrenia and psychosis (van Rossum et al, 2011).

In addition to the effect in the dmPFC, we also found group by drug interactions in the superior frontal gyrus and occipital areas. Similar to the pattern seen in the dmPFC, psychosis subjects showed a reversal or absence of the tendency of LRZ to reduce BOLD signal in control subjects. Some of these areas correspond to regions elicited by emotionally salient stimuli (except for the superior frontal gyrus (Kober et al, 2008)). The postmortem work has tended to focus on frontal cortical regions, particularly the dorsolateral PFC and the ACC, but similar findings have been noted in the occipital cortex (Hashimoto et al, 2008b), suggesting that GABAergic abnormalities are more generalized in the schizophrenia cortex. Our results are consistent with this idea of more widespread GABAergic abnormalities.

The only main effects of LRZ appeared in secondary visual cortex and cerebellum, reducing BOLD signal to stimulation. Although the psychosis patients showed significantly less reduction than the comparison subjects in the left mid-occipital gyrus, consistent with postmortem findings described above, the patients also showed signal reduction with LRZ, particularly in right occipital cortex. Reduced BOLD signal in occipital cortex is consistent with inhibitory effects of LRZ on aggregate neuronal activity, as GABAergic mechanisms have been implicated in control of the aggregate BOLD signal in visual cortex (Qin et al, 2012). Inhibition of occipital cortex has been previously reported after GABAR potentiation (Menzies et al, 2007). The appearance of main effects of LRZ only in posterior regions may reflect various factors such as differing local populations of GABAR as well as the dynamic conditions of local circuitry engaged in the task with strong occipital stimulation. LRZ, as a non-subtype selective modulator of the GABAR, can have multiple effects on neural function measured with BOLD. Future research with subtype selective agents may provide more information about subunitspecific effects of stimulation, which would be important to differentiate activity, eg, $\alpha-1$ from $\alpha-2$ GABAR subunits in psychosis.
Several of our hypotheses were not supported. Contrary to predictions about a valence-specific effect of negative stimuli, we found no valence by group by drug interactions, neither in the dmPFC nor other regions throughout the brain. All of the significant effects were driven by the more robust signal given by contrasts of pictures with the implicit baseline. In retrospect, the lack of significant interactions is not surprising, given that the neutral stimuli were themselves quite salient, with social content that is processed in the MFC (Amodio and Frith, 2006). Our task was focused on experiencing, not labeling, the stimuli, and imaging studies of emotional experience in schizophrenia have demonstrated relatively few differences from controls in contrast to labeling emotional expressions (Taylor et al, 2012). Within the MFC, we did not find abnormal activity of the ACC that has been implicated in emotion studies of schizophrenia (Taylor et al, 2012). Although functionally coupled with the overlaying dmPFC (Kober et al, 2008), the lack of a strong response component to our task may have been the reason we did not see a signal in the ACC. We also failed to find a significant signal in the aIns, although Figure 3 shows that sub-threshold activity occurred in the region of the right aIns, just outside our a priori search area. Thus, the failure to confirm this hypothesis may represent a Type 2 error.

Several caveats and limitations should be kept in mind. The sample size is small, although the two groups were tightly matched, and the within-subjects design maximized power to detect drug effects. All but one of the 14 patients were taking antipsychotic medications, which, while very unlikely to cause the observed correlations, remain a potential confound in the group by drug effects, even though correlations with antipsychotic dosage were absent. It is worth noting that chronic exposure to haloperidol does not change GABAR transcript expression in monkey cortex (Hashimoto et al, 2008a). We did not measure LRZ blood levels, although the intravenous infusion paradigm and dosing based on body weight avoided individual differences because of differential absorption rates of orally ingested medications used in other LRZ challenge studies (Menzies et al, 2007). As sedation is a common side effect of LRZ, the experiment was designed to minimize this potential confound, and the failure of subjects (as a group) to successfully guess when they had the LRZ infusion suggests that we were successful in minimizing subjective effects of LRZ. The viewing task elicited a wide range of response latencies (in contrast to a more controlled cognitive task) that may have reflected some inattentiveness to the low task demands, but behavior differed neither between groups nor with drug infusion, suggesting that poor attention could not explain our main results.

In summary, the results of this study suggest that abnormal GABAergic activity occurs in schizophrenia, involving negative affect on dimension extending into non-ill populations. Although prior work on GABAergic dysfunction focused on the presumed role of these postmortem abnormalities on cognitive function (Lewis et al, 2012; Menzies et al, 2007), work presented here suggests that negative affect is an alternative process to consider when correlating GABAergic mechanisms with behavior, with implications for designing treatment interventions. 


\section{FUNDING AND DISCLOSURE}

This work was supported the National Institute of Mental Health (R21MH086701), the Boledovich Schizophrenia Research Fund, and University of Michigan Clinical Translational Science Award (UL1RR024986). SFT has a research contract with St Jude Medical and research support from Neuronetics. The authors declare no conflict of interest.

\section{ACKNOWLEDGEMENTS}

We acknowledge the assistance of Inga Brege for subject recruitment, data acquisition, and workup.

\section{REFERENCES}

Abi-Dargham A, Laruelle M, Krystal J, D'Souza C, Zoghbi S, Baldwin RM et al (1999). No evidence of altered in vivo benzodiazepine receptor binding in schizophrenia. Neuropsychopharmacology 20: 650-661.

Addington D, Addington J, Maticka-Tyndale E (1993). Assessing depression in schizophrenia: the Calgary Depression Scale. $\mathrm{Br} J$ Psychiatry Suppl 163: 39-44.

Ahn K, Gil R, Seibyl J, Sewell RA, D’Souza DC (2011). Probing GABA receptor function in schizophrenia with iomazenil. Neuropsychopharmacology 36: 677-683.

American Psychiatric Association (1994). Diagnostic and Statistical Manual of Mental Disorders. 4th edn. (DSM-IV) American Psychiatric Association: Washington, DC, USA.

Amodio DM, Frith CD (2006). Meeting of minds: the medial frontal cortex and social cognition. Nat Rev Neurosci 7: 268-277.

Andreasen NC (1983). The scale for the Assessment of Negative Symptoms. The University of Iowa: Iowa City.

Bartos M, Vida I, Jonas P (2007). Synaptic mechanisms of synchronized gamma oscillations in inhibitory interneuron networks. Nat Rev Neurosci 8: 45-56.

Carpenter WT Jr., Buchanan RW, Kirkpatrick B, Breier AF (1999). Diazepam treatment of early signs of exacerbation in schizophrenia. Am J Psychiatry 156: 299-303.

Cohen AS, Minor KS (2010). Emotional experience in patients with schizophrenia revisited: meta-analysis of laboratory studies. Schizophr Bull 36: 143-150.

Cohen S, Kamarck T, Mermelstein R (1983). A global measure of perceived stress. J Health Soc Behav 24: 385-396.

Cousins DA, Young AH (2007). The armamentarium of treatments for bipolar disorder: a review of the literature. Int J Neuropsychopharmacol 10: 411-431.

Daskalakis ZJ, Christensen BK, Chen R, Fitzgerald PB, Zipursky RB, Kapur S (2002). Evidence for impaired cortical inhibition in schizophrenia using transcranial magnetic stimulation. Arch Gen Psychiatry 59: 347-354.

Etkin A, Egner T, Kalisch R (2011). Emotional processing in anterior cingulate and medial prefrontal cortex. Trends Cogn Sci 15: 85-93.

First MB, Spitzer RL, Gibbon M, Williams J (1995). Structured Clinical Interview for DSM-IV Axis I Disorders, Patient Edition (SCID-P), ver 2.0. Biometrics Research: New York, USA.

Fredrickson BL, Tugade MM, Waugh CE, Larkin GR (2003). What good are positive emotions in crises? A prospective study of resilience and emotions following the terrorist attacks on the United States on September 11th, 2001. J Pers Soc Psychol 84: 365-376.

Guidotti A, Auta J, Davis JM, Di-Giorgi-Gerevini V, Dwivedi Y, Grayson DR et al (2000). Decrease in reelin and glutamic acid decarboxylase67 (GAD67) expression in schizophrenia and bipolar disorder: a postmortem brain study. Arch Gen Psychiatry 57: 1061-1069.
Hashimoto T, Arion D, Unger T, Maldonado-Aviles JG, Morris HM, Volk DW et al (2008a). Alterations in GABA-related transcriptome in the dorsolateral prefrontal cortex of subjects with schizophrenia. Mol Psychiatry 13: 147-161.

Hashimoto T, Bazmi HH, Mirnics K, Wu Q, Sampson AR, Lewis DA (2008b). Conserved regional patterns of GABA-related transcript expression in the neocortex of subjects with schizophrenia. Am J Psychiatry 165: 479-489.

Hollingshead AB, Redlich FC (1958). Social class and mental illness. Am J Psychiatry 149: 1035-1044.

Horan WP, Blanchard JJ, Clark LA, Green MF (2008). Affective traits in schizophrenia and schizotypy. Schizophr Bull 34: 856-874.

Jastak S, Wilkinson GS (1984). Wide Range Achievement Test Revised. Jastak Associates, Inc.: Wilmington, DE, USA.

Jenkinson M, Bannister P, Brady M, Smith S (2002). Improved optimization for the robust and accurate linear registration and motion correction of brain images. Neuroimage 17: 825-841.

Keefe RS, Goldberg TE, Harvey PD, Gold JM, Poe MP, Coughenour L (2004). The brief assessment of cognition in schizophrenia: reliability, sensitivity, and comparison with a standard neurocognitive battery. Schizophr Res 68: 283-297.

Kegeles LS, Mao X, Stanford AD, Girgis R, Ojeil N, Xu X et al (2012). Elevated prefrontal cortex gamma-aminobutyric acid and glutamate-glutamine levels in schizophrenia measured in vivo with proton magnetic resonance spectroscopy. Arch Gen Psychiatry 69: 449-459.

Kober H, Barrett LF, Joseph J, Bliss-Moreau E, Lindquist K, Wager TD (2008). Functional grouping and cortical-subcortical interactions in emotion: a meta-analysis of neuroimaging studies. Neuroimage 42: 998-1031.

Kring AM, Moran EK (2008). Emotional response deficits in schizophrenia: insights from affective science. Schizophr Bull 34: 819-834.

Lewis DA, Curley AA, Glausier JR, Volk DW (2012). Cortical parvalbumin interneurons and cognitive dysfunction in schizophrenia. Trends Neurosci 35: 57-67.

Menzies L, Ooi C, Kamath S, Suckling J, McKenna P, Fletcher P et al (2007). Effects of gamma-aminobutyric acid-modulating drugs on working memory and brain function in patients with schizophrenia. Arch Gen Psychiatry 64: 156-167.

Nakazawa K, Zsiros V, Jiang Z, Nakao K, Kolata S, Zhang S et al (2012). GABAergic interneuron origin of schizophrenia pathophysiology. Neuropharmacology 62: 1574-1583.

Olsen RW, Tobin AJ (1990). Molecular biology of GABAA receptors. Faseb J 4: 1469-1480.

Overall JE, Gorham DR (1962). Brief psychiatric rating scale. Psychol Rep 10: 799-812.

Paulus MP, Feinstein JS, Castillo G, Simmons AN, Stein MB (2005). Dose-dependent decrease of activation in bilateral amygdala and insula by lorazepam during emotion processing. Arch Gen Psychiatry 62: 282-288.

Phillips LK, Seidman LJ (2008). Emotion processing in persons at risk for schizophrenia. Schizophr Bull 34: 888-903.

Qin P, Duncan NW, Wiebking C, Gravel P, Lyttelton O, Hayes DJ et al (2012). GABA(A) receptors in visual and auditory cortex and neural activity changes during basic visual stimulation. Front Hum Neurosci 6: 337.

Spielberger CD, Gorsuch RL, Lushene R, Vagg PR, Jacobs GA (1983). Manual for the State-Trait Anxiety Inventory (Form Y). Mind Garden: Palo Alto, CA, USA.

Szabadics J, Varga C, Molnar G, Olah S, Barzo P, Tamas G (2006). Excitatory effect of GABAergic axo-axonic cells in cortical microcircuits. Science 311: 233-235.

Taylor SF, Chen AC, Tso IF, Liberzon I, Welsh RC (2011). Social appraisal in chronic psychosis: role of medial frontal and occipital networks. J Psychiatr Res 45: 526-538.

Taylor SF, Kang J, Brege IS, Tso IF, Hosanagar A, Johnson TD (2012). Meta-analysis of functional neuroimaging studies of 
GABAergic processing in schizophrenia SF Taylor et al

emotion perception and experience in schizophrenia. Biol Psychiatry 71: 136-145.

Taylor SF, Welsh RC, Chen AC, Velander AJ, Liberzon I (2007). Medial frontal hyperactivity in reality distortion. Biol Psychiatry 61: 1171-1178.

Tso IF, Grove TB, Taylor SF (2010). Emotional experience predicts social adjustment independent of neurocognition and social cognition in schizophrenia. Schizophr Res 122: 156-163.

Tzourio-Mazoyer N, Landeau B, Papathanassiou D, Crivello F, Etard O, Delcroix N et al (2002). Automated anatomical labeling of activations in SPM using a macroscopic anatomical parcellation of the MNI MRI single-subject brain. Neuroimage 15: 273-289.

van Rossum I, Dominguez MD, Lieb R, Wittchen HU, van Os J (2011). Affective dysregulation and reality distortion: a 10-year prospective study of their association and clinical relevance. Schizophr Bull 37: 561-571.

Volk DW, Pierri JN, Fritschy JM, Auh S, Sampson AR, Lewis DA (2002). Reciprocal alterations in pre- and postsynaptic inhibitory markers at chandelier cell inputs to pyramidal neurons in schizophrenia. Cereb Cortex 12: 1063-1070.

Wassef AA, Dott SG, Harris A, Brown A, O’Boyle M, Meyer WJ 3rd et al (1999). Critical review of GABA-ergic drugs in the treatment of schizophrenia. J Clin Psychopharmacol 19: 222-232.
Wermeling DP, Miller JL, Archer SM, Manaligod JM, Rudy AC (2001). Bioavailability and pharmacokinetics of lorazepam after intranasal, intravenous, and intramuscular administration. J Clin Pharmacol 41: 1225-1231.

Wise RG, Lujan BJ, Schweinhardt P, Peskett GD, Rogers R, Tracey I (2007). The anxiolytic effects of midazolam during anticipation to pain revealed using fMRI. Magn Reson Imaging 25: 801-810.

Woodruff A, Xu Q, Anderson SA, Yuste R (2009). Depolarizing effect of neocortical chandelier neurons. Front Neural Circuits 3: 15.

Woods SW (2003). Chlorpromazine equivalent doses for the newer atypical antipsychotics. J Clin Psychiatry 64: 663-667.

Worsley KJ, Friston KJ (1995). Analysis of fMRI time-series revisited-again. Neuroimage 2: 173-181.

Yang Y, Gu H, Zhan W, Xu S, Silbersweig D, Stern E (2002). Simultaneous perfusion and BOLD imaging using reverse spiral scanning at 3T: characterization of functional contrast and susceptibility artifacts. Magn Reson Med 48: 278-289.

Yoon JH, Maddock RJ, Rokem A, Silver MA, Minzenberg MJ, Ragland JD et al (2010). GABA concentration is reduced in visual cortex in schizophrenia and correlates with orientation-specific surround suppression. J Neurosci 30: 3777-3781.

Supplementary Information accompanies the paper on the Neuropsychopharmacology website (http://www.nature.com/npp) 